\title{
Distribution of Internal Elastic Lamina and External Elastic Lamina in the Internal Carotid Artery: Possible Relationship With Atherosclerosis
}

\author{
Toru MASUOKA, Nakamasa HAYASHI, Emiko HORI, \\ Naoya KUWAYAMA, Osamu OHTANI*, and Shunro ENDO
}

Departments of Neurosurgery and * Anatomy, Faculty of Medicine, University of Toyama, Toyama

\begin{abstract}
The intracranial internal carotid artery (ICA) is a muscular artery and lacks external elastic lamina (EEL). Stenosis of the intracranial ICA is relatively uncommon, but the most common site is the cavernous portion. The characteristics of the arterial wall structures were examined using serial 3-mm sections of 32 intracranial ICAs obtained from 50 cadavers to identify where the EEL disappeared. The portions of the ICA where the intima exhibited thickening were also determined. Both the internal elastic lamina (IEL) and EEL were observed in all 32 specimens of the petrous portion of the ICA. Only the IEL was observed in all 32 specimens of the intradural portion of the ICA. The EEL had disappeared in 31 of the 32 specimens of the horizontal segment of the cavernous portion of the ICA. Intimal thickening of the ICA was observed in 23 of 32 ICA specimens, and frequently appeared in the horizontal segment of the cavernous portion of the ICA. The EEL disappeared in the cavernous portion of the ICA, which is the most common site of stenosis of the intracranial ICA. Change in the elasticity of the arterial wall in the cavernous portion may be an important factor in the process of atherosclerosis in the intracranial ICA.
\end{abstract}

Key words: atherosclerosis, cavernous sinus, external elastic lamina, internal carotid artery, internal elastic lamina

\section{Introduction}

Atherosclerotic stenosis of the internal carotid artery (ICA) is common, and most frequently occurs in the cervical portion of the ICA just beyond its origin. Stenosis of the intracranial ICA is less common, and is usually located between the carotid canal and the origin of the ophthalmic artery. ${ }^{6)}$ The most common site of stenosis of the intracranial ICA is the cavernous portion. ${ }^{3)}$ The causes of development of atheromatous plaque around the carotid bifurcation have been frequently investigated.1,15,21) However, the special features marking atherosclerotic change in the cavernous portion of the ICA are poorly understood.

There are two types of arteries, muscular arteries and elastic arteries, which differ primarily in the tunica media. The tunica media of elastic arteries consists mainly of elastic fibers, whereas that of muscular arteries consists mainly of smooth muscle cells. Muscular arteries have two elastic layers in the

Received June 2, 2009; Accepted August 4, 2009 tunica media, the internal elastic lamina (IEL) and the external elastic lamina (EEL). The common carotid artery is an elastic artery, whereas the ICA is a muscular artery, and the change occurs at the carotid bifurcation. ${ }^{7,8)}$ The intracranial ICA is characterized by the lack of an EEL, ${ }^{10,11)}$ and the elastic fibers are concentrated inside the IEL. ${ }^{5,17)}$ Microscopic examination of the arterial wall in the cavernous and petrous portions of the ICA showed that the EEL is present in the petrous portion of the ICA but disappears within the cavernous portion. ${ }^{13)}$

The present study examined the characteristics of the arterial wall structures in detail using serial sections of the intracranial ICA to investigate the relationship between atherosclerotic stenosis and arterial wall structures in the cavernous portion of the ICA.

\section{Method}

Thirty-two specimens of the intracranial ICA were obtained from 50 cadavers, without knowledge of their causes of death or medical history. The 


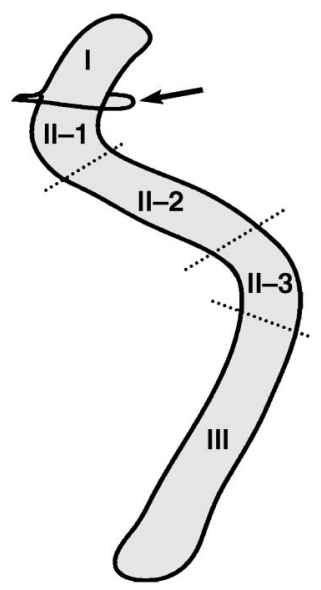

Fig. 1 Schematic drawing of the anatomical classification of the internal carotid artery: $I$, intradural portion; II, cavernous portion (II-1, anterior knee; II-2, horizontal segment; II-3, posterior knee); III, petrous portion. Arrow indicates dural ring.

patients were aged 57 to 100 years at the time of death (mean 82.0 years). Serial sections of specimens were obtained at $3-\mathrm{mm}$ intervals and stained using the Elastica van Gieson method. The ICA was classified into the intradural portion (I), cavernous portion (II), and petrous portion (III) (Fig. 1). We considered the portion distal to the dural ring to comprise the intradural ICA. The cavernous portion was subdivided into the anterior knee (II-1), horizontal segment (II-2), and posterior knee (II-3). Histological sections were examined under a light microscope at $400 \times$ magnification. The presence of the EEL in the wall of the ICA was evaluated. Regions of the ICA with thick intima were also determined.

\section{Results}

Figure 2 shows representative photomicrographs of various portions of the intracranial ICA. The IEL was observed in all segments of all 32 specimens. The EEL was observed in all 32 specimens of the petrous portion (III) of the ICA and in 31 specimens of the posterior knee (II-3) (Fig. 3). In contrast, the EEL was present in 19 specimens of the proximal portion and 12 specimens of the distal portion of the horizontal segment (II-2), in 0 specimens of the anterior knee (II-1), and 0 specimens of the intradural segment (I) (Fig. 3). Thick intima was recognized in the posterior knee (II-3) in 2 specimens, the proximal portion of the horizontal segment in 12 specimens, and the distal portion of the horizontal segment in 9 specimens.

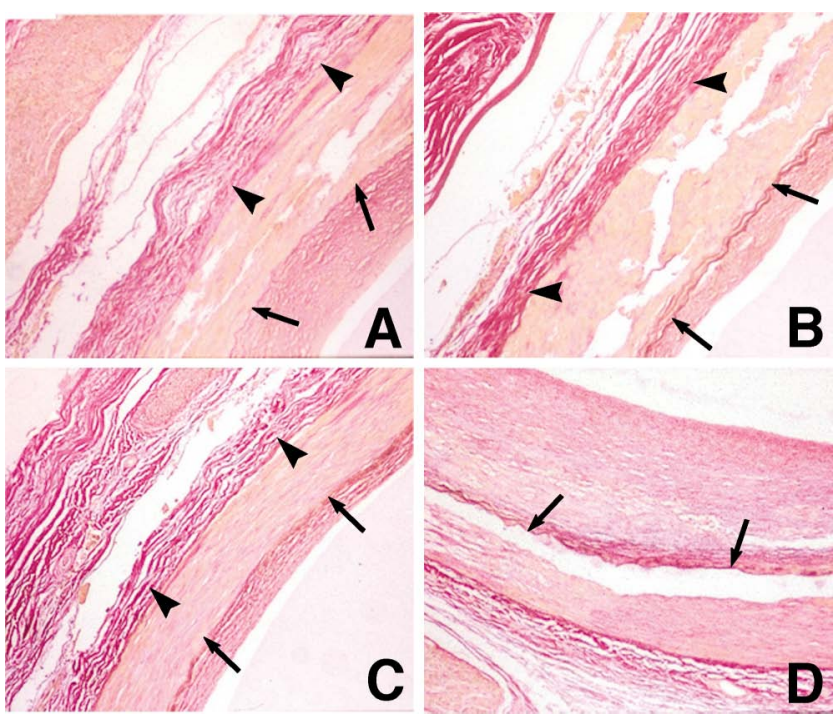

Fig. 2 Photomicrographs of various portions of internal carotid artery (ICA). Elastica van Gieson stain, $\times$ 400. A: Petrous portion of the ICA. Note that both internal elastic lamina (IEL: arrows) and external elastic lamina (EEL: arrowheads) are present. B: Posterior knee. C: Proximal horizontal segment of the cavernous portion. D: Distal horizontal segment of the cavernous portion. Note that the IEL is present, but not the EEL.

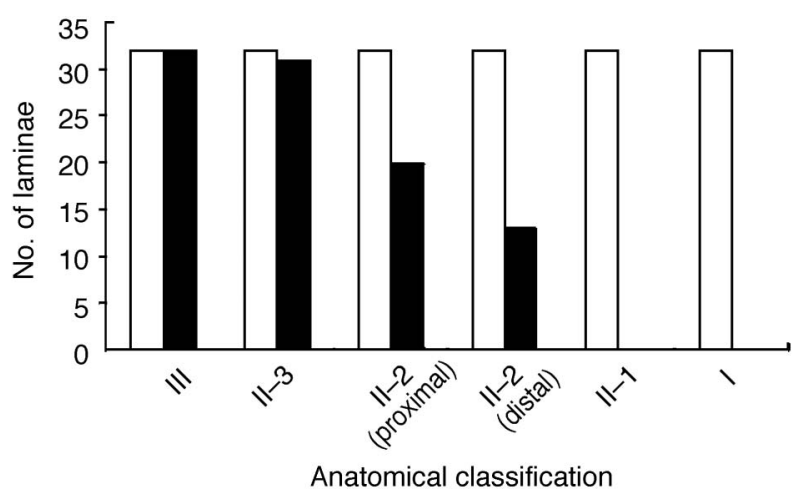

Fig. 3 Presence of internal elastic lamina (open columns) and external elastic lamina (closed columns). See Fig. 1 for anatomical classification.

\section{Discussion}

The previous detailed study of the extradural and intradural portions of the intracranial ICA observed that the adventitia was thicker in the intracranial extradural carotid segment than in the intradural segment. ${ }^{13)}$ The EEL, located between the collagen fibers of the adventitia and the muscle coat of the media, was a well-defined structure in the petrous portion of the intracranial ICA, but disappeared wi- 
thin the cavernous portion of the ICA. The EEL was not observed in the intradural segments of the ICA. The present study of the arterial wall structures in serial sections of the intracranial ICA found that the EEL disappeared in the horizontal segment of the cavernous portion of the ICA. Marked reduction or loss of elastic fibers in the tunica media and EEL is also observed in the portion of the vertebral artery entering the skull.4,19) This change is most pronounced in the last $0.5 \mathrm{~cm}$ extradurally, but is not complete until about $0.5 \mathrm{~cm}$ beyond the point of dural perforation. ${ }^{19)}$

Intracranial atherosclerotic stenosis typically occurs in the petrous cavernous siphon segments of the ICA. ${ }^{20)}$ The North American Carotid Symptomatic Carotid Artery Trial found that the infraclinoid portion of the ICA was affected 7 times more often than the supraclinoid portion. ${ }^{9)}$ Examination of the prevalence of intracranial cerebral artery stenosis in patients with extracranial carotid artery stenosis showed that the petrocavernous segment of the ICA was the most commonly involved site. ${ }^{2)}$ The interosseous course of the petrous portion of the ICA is a unique feature, but stenotic lesions are least common in this segment of ICA. ${ }^{14)}$ The cavernous portion of the ICA is the most common site of atherosclerotic stenosis of the intracranial ICA. ${ }^{3)}$ Stenosis of the intracranial ICA was found in the cavernous portion in $72 \%$ of cases, in the petrous portion in $16 \%$, and in the intradural ICA in $12 \%{ }^{3)}$ Atherosclerotic stenosis of the intracranial ICA occurs in the petrous portion in $7 \%$ of cases, in the cavernous portion in $68 \%$, in the intradural ICA in $48 \%$, and at multiple sites in $23 \% .^{12)}$

The present study identified regions of the intracranial ICA with thick intima, often referred to as early progression of atherosclerosis, ${ }^{18)}$ are most frequently occurring in the horizontal segment of the cavernous portion of the ICA. The tortuosity of the cavernous portion of the ICA and associated flow characteristics, as well as the composition of the vessel wall in this area, have been suggested to be factors predisposing to atherosclerosis. ${ }^{12,13,16)}$

The present study found that the EEL disappeared in the cavernous portion of the ICA, which is the most common site of stenosis of the intracranial ICA. Change in the elasticity of the arterial wall in the cavernous portion may be an important factor in the process of atherosclerosis in the intracranial ICA.

\section{References}

1) Affeld K, Goubergritis L, Fernandez-Britto J, Falcon L: Variability of the geometry of the human common carotid artery. A vessel cast study of 31 specimens. Pathol Res Pract 194: 597-602, 1998

2) Alkan O, Kizilkilic O, Yildirim T, Atalay H: Intracranial cerebral artery stenosis with associated coronary artery and extracranial carotid artery stenosis in Turkish patients. Eur J Radiol 71: 450-455, 2009

3) Craig DR, Meguro K, Watridge C, Robertson JT, Barnett HJM, Fox AJ: Intracranial internal carotid stenosis. Stroke 13: 825-828, 1982

4) Fang H: A comparison of blood vessels of the brain and peripheral blood vessels, in Wright IS, Millikan CH (eds): Cerebral Vascular Diseases. New York, Grune \& Stratton, 1958, pp 17-22

5) Fujimoto K: 'Medial defects' in the prenatal human cerebral arteries. Stroke 27: 706-708, 1996

6) Haas WK, Fields WS, North RR, Kricheff II, Chase NE, Bauer RB: Joint Study of Extracranial Arterial Occlusion: II. Arteriography techniques, sites, and complications. JAMA 203: 961-968, 1968

7) Hori E, Hayashi N, Hamada H, Masuoka T, Kuwayama N, Hirashima Y, Origasa H, Ohtani O, Endo S: A development of atheromatous plaque is restricted by characteristic arterial wall structure at the carotid bifurcation. Surg Neurol 69: 586-591, 2008

8) Janzen J, Lanzer P, Rothenberger-Janzen K, Vuong PN: Variable extension of the transitional zone in the medial structure of carotid artery tripod. Vasa 30: 101-106, 2001

9) Kappelle LJ, Eliasziw M, Fox AJ, Sharpe BL, Barnett $\mathrm{HJ}$ : Importance of intracranial atherosclerotic disease in patients with symptomatic stenosis of the internal carotid artery. Stroke 30: 282-286, 1999

10) Kondo S, Hashimoto N, Kikuchi H, Hazama F, Nagata I, Kataoka H: Cerebral aneurysms arising at nonbranching sites. Stroke 28: 398-404, 1997

11) Kondo S, Hashimoto N, Kikuchi H, Hazama F, Nagat I, Kataoka H: Apoptosis of medial smooth muscle cells in the development of saccular cerebral aneurysms in rats. Stroke 29: 181-189, 1998

12) Raju S, Fredericks RK: Carotid siphon stenosis. J Cardiovasc Surg 28: 671-677, 1987

13) Ratinov G: Extradural intracranial portion of carotid artery. Arch Neurol 10: 66-73, 1964

14) Roederer GO, Langlois YE, Chan AR, Chikos PM, Thiele BL, Strandness DE Jr: Is siphon disease important in predicting outcome of carotid endarterectomy? Arch Surg 118: 1177-1181, 1983

15) Schulz UG, Rothwell PM: Major variation in carotid bifurcation anatomy. A possible risk factor for plaque development? Stroke 32: 2522-2529, 2001

16) Schwartz CJ, Mitchell JRA: Observations on localization of arterial plaque. Circ Res 11: 63-73, 1962

17) Stehben WE: Cerebrovascular disease, in Stehbens WE, Lie LT (eds): Vascular Pathology. London, Tokyo, Chapman and Hall, 1995, pp 437-488

18) Vazquez-Padron RI, Lasko D, Li S, Louis L, Pestana IA, Pang M, Liotta C, Fornoni A, Aitouche A, Pham SM: Aging exacerbates neointimal formation, and increases proliferation and reduces susceptibility to 
apoptosis of vascular smooth muscle cells in mice. J Vasc Surg 40: 1199-1207, 2004

19) Wilkinson IMS: The vertebral artery. Extracranial and intra-cranial structure. Arch Neurol 27: 392-396, 1972

20) Wojak JC, Dunlap DC, Hargrave KR, DeAlvare LA, Culbertson HS, Connors JJ III: Intracranial angioplasty and stenting: long-term results from a single center. AJNR Am J Neuroradiol 27: 1882-1892, 2006

21) Zarins CK, Giddens DP, Bharadvaj BK, Sottiurai VS, Mabon RF, Glagov S: Carotid bifurcation atherosclerosis. Quantitative correlation of plaque localization with flow velocity profiles and wall shear stress. Circ Res 53: 502-514, 1983

Address reprint requests to: Nakamasa Hayashi, M.D., Department of Neurosurgery, Faculty of Medicine, University of Toyama, 2630 Sugitani, Toyama 930-0194, Japan.

e-mail: nakamasa@iwa.att.ne.jp

\section{Commentary}

This simple but elegant pathoanatomic study by Masuoka et al. provides interesting and novel observations regarding the loss of external elastic lamina (EEL) in the horizontal cavernous segment of the internal carotid artery (ICA). Long recognized to lack EEL in the subarachnoid space, it has not been previously noted, to my knowledge, that EEL is lost in the cavernous segment and not more distally at the transition into the subarachnoid space.

The authors also note intimal thickening in the cavernous segment, they point to the prevalence of intracranial stenosis in that segment, and speculate that atherogenesis may be related to the loss of elasticity in that segment associated with loss of EEL. We caution, as they do, that this association does not prove causation, and other factors than loss of EEL, including unique hemodynamic stresses may be involved in atherogenesis vulnerability in that location. Other carotid locations such as the cervical carotid bifurcation are quite prone to atherogenesis without loss of EEL, and more distal intracranial arteries lack EEL and have infrequent atheromas.

The loss of EEL in the cavernous segment may also explain the vulnerability of this segment, but not the more proximal ICA, to the development of saccular aneurysms, which luckily are unlikely to bleed because they are not in the subarachnoid space, but they occur nevertheless. And the loss of EEL (along with the absence of surrounding arachnoid layer) may explain in part the vulnerability of the cavernous segment to vessel injury when peeling tumors away from it, as opposed to the more proximal ICA which seems more resilient (or more distal arteries where an arachnoid plane often helps).

The authors are congratulated on their careful observations which should motivate novel hypotheses related to several cerebrovascular pathologies.

Issam A. AwAD, M.D., M.Sc., F.A.C.S., M.A. (Hon.) Neurovascular Surgery Program Section of Neurosurgery University of Chicago Medical Center Chicago, Illinois, U.S.A.

This paper provides a convincing and rational explanation for a fact of common observation, i.e. the relatively high incidence of atheromatic plaques in the horizontal segment of the cavernous carotid artery. The authors correlate the presence of intimal thickening and/or of atheromatic changes to the microscopic demonstration of a decreased elastic component of the vessel wall.

It explains also why the most frequent localization of atheroma is at the level of the carotid bifurcation in the neck: the authors point out that the transition from an elastic vessel, such as the common carotid artery, to a muscular vessel (typically with less elastic material in the tunica media) such as the internal carotid artery, can explain the stress on the intima, that in turn leads to a stenotic modification of the wall.

In conclusion, this is a simple work, but useful to read in order to increase the understanding of the pathology.

Alessandro Ducati, M.D. Ordinario di Neurochirurgia

Universita' di Torino Torino, Italy 